\title{
Über das Vergleichen: Komparative Forschung in deutschen kommunikationswissenschaftlichen Fachzeitschriften 1948-2005
}

\author{
Thomas Hanitzsch/Klaus-Dieter Altmeppen
}

Die vorliegende Studie ziebt eine inhaltsanalytische Bilanz aus mebr als einem halben Jahrbundert ländervergleichender Forschung in den drei fübrenden deutschen kommunikationswissenschaftlichen Fachzeitschriften Medien \& Kommunikationswissenschaft (Rundfunk und Fernsehen), Publizistik und Media Perspektiven. Dabei offenbart sich - trotz eines positiven Trends, der in den 1990er Jabren einsetzt - ein größerer Nachbolbedarf im Hinblick auf die Beachtung grundlegender Qualitätsstandards. So liefern die meisten Studien keinerlei Begründung für das komparative Vorgeben und die Auswabl der Länder. Zudem ist die vergleichende Forschung nur ungenügend in eine theoretische Perspektive eingebettet, der größte Teil der untersuchten Arbeiten ist atheoretischer Natur. Zu wichtigen Qualitätsstandards wie der Äquivalenz von Datenerhebungsmethoden, Forschungsinstrumenten, Erbebungszeiträumen, Grundgesamtheiten und Verfabren der Fallauswabl feblen oftmals hinreichende Angaben.

Schlagwörter: Komparative Forschung, methodologische Standards, Inhaltsanalyse

\section{Zur komparativen Forschung und zu diesem Beitrag}

Nachdem die komparative Forschung in der deutschen Medien- und Kommunikationswissenschaft lange Zeit nur ein Schattendasein führte (vgl. Kleinsteuber 2003: 78), erfreut sie sich in den letzten Jahren zunehmender Beliebtheit. Das Ende des Kalten Krieges und voranschreitende Globalisierungsprozesse haben die Mobilitätschancen für Wissenschaftler erheblich erhöht und eine Vielzahl von Gelegenheiten für den internationalen Austausch zwischen Fachkollegen geschaffen. Die rasante Entwicklung der Informationstechnologien verkürzt Kommunikationswege, wodurch die Abstimmung in internationalen Forschernetzwerken enorm erleichtert wird. Darüber hinaus hat auch in den Einrichtungen der Wissenschaftsförderung mittlerweile ein Umdenken eingesetzt, wodurch transnationale Verbundprojekte heute bessere Aussichten auf eine Finanzierung haben als jemals zuvor.

Komparative Studien bilden einen zentralen Forschungsbereich innerhalb der Medien- und Kommunikationswissenschaft. Deutlich wird dies nicht zuletzt in der rasant zunehmenden Zahl von einschlägigen Publikationen (vgl. u. a. Blumler, McLeod \& Rosengren 1992; Esser \& Pfetsch 2003) und Konferenzen, die sich diesem Thema explizit und zum Teil sogar exklusiv annehmen. ${ }^{1}$ Der Bedeutungszuwachs von komparativen Forschungsansätzen wird dabei in letzter Zeit von einer verstärkten Institutionalisierung begleitet. Das im Jahr 2006 erstmals erschienene Journal of Intercultural Communication Research steht in der Tradition ähnlicher Zeitschriften aus anderen Disziplinen, wie das International E Comparative Law Quarterly (gegründet 1952), die Compara-

1 Vgl. u. a. die ICA-Pre-Konferenz „Methodologies of Comparative Media Research in a Global Sphere: Paradigms - Critique - Methods“ in San Francisco, Mai 2007. 
tive Studies in Society and History (1958), Comparative Politics (1968) und das Journal of Cross-Cultural Psychology (1970).

Darüber hinaus sind allein im deutschsprachigen Raum mehrere Lehrstühle mit einem Schwerpunkt auf vergleichende Medien- und Kommunikationsforschung besetzt worden, u. a. an den Universitäten Bochum, Erfurt und Zürich. An der Österreichischen Akademie der Wissenschaften ist eine Kommission für vergleichende Medien- und Kommunikationsforschung tätig, und an der Universität Luzern wurde ein Master-Studiengang in „Vergleichender Medienwissenschaft“ eingerichtet. Ähnliche Programme werden schon seit einigen Jahren von verschiedenen Universitäten im angelsächsischen Raum angeboten: zum Beispiel Studiengänge in „Comparative Media Studies“ am Massachusetts Institute of Technology, „Comparative Media Law \& Policy“ an der University of Oxford sowie „Comparative Journalism“ an der University of Wales.

In der Medien- und Kommunikationswissenschaft wurde die zunehmende Bedeutung der komparativen Forschung allerdings nicht immer von einer adäquaten Weiterentwicklung des theoretischen und methodologischen Arsenals begleitet. Während in benachbarten Wissenschaftsdisziplinen bereits eine Fülle von grundlegenden und einführenden Standardwerken vorliegt (vgl. für die Politikwissenschaft: Landman 2000 sowie Przeworski \& Teune 1970; Psychologie: van de Vijver \& Leung 1997; Soziologie: Kohn 1989b und Øyen 1990), befindet sich die Diskussion über Designs und Methoden international vergleichender Studien in unserem Fach noch weitgehend am Anfang. In letzter Zeit ist jedoch ein Trend zu methodologischer Aufarbeitung zu verzeichnen, und eine Entwicklung von Qualitätsstandards hat bereits eingesetzt (vgl. Chang et al. 2001; Jensen 1998; Johnson \& Tuttle 2000; Livingstone 2003; Wirth \& Kolb 2003). Darüber hinaus ist festzustellen, dass die komparative Forschung stärker deskriptiv und weniger explanativ angelegt ist, womit sie „eher farbenreiche Beschreibungen produziert als Erklärungen wichtiger Probleme" (Peter 2003: 191).

Vor dem Hintergrund des schnell wachsenden Erfahrungsschatzes im Hinblick auf die Planung, Durchführung und Verwertung kulturvergleichender Forschung will der vorliegende Aufsatz das deutsche Spektrum komparativer Studien einer inhaltsanalytischen Bilanz unterziehen sowie die Entwicklung in diesem Feld über fast sechs Jahrzehnte hinweg abbilden. Die Meta-Untersuchung ließ sich dabei von der zentralen Frage leiten, inwieweit die Qualitätsstandards der komparativen Forschung in Beiträgen deutscher Fachzeitschriften Berücksichtigung finden bzw. fanden. Datengrundlage ist eine Vollerhebung der drei bedeutendsten kommunikations- und medienwissenschaftlichen Fachorgane im deutschsprachigen Raum: Medien E Kommunikationswissenschaft (früher: Rundfunk und Fernsehen, 1948-2005), Publizistik (1956-2005) und Media Perspektiven (1970-2005). Im Folgenden sollen zunächst die theoretischen Grundlagen der Untersuchung sowie das methodische Vorgehen dargestellt werden. Danach werden die wichtigsten Befunde der quantitativen und qualitativen Inhaltsanalyse präsentiert und einer Bewertung unterzogen.

\section{Grundlagen komparativer Forschung}

Die Wurzeln der vergleichenden Forschung lassen sich zum Werk des englischen Gelehrten Edward Tylor zurückverfolgen, dessen Buch „Primitive Culture“ (1871) einen Meilenstein in der englischsprachigen Kulturanthropologie bildet. Die erste groß angelegte Vergleichsstudie war gegen Ende des 19. Jahrhunderts vermutlich Emil Durkheims (1897/1993) Untersuchung von Selbstmordraten in unterschiedlichen Gesellschaften. Dennoch verstrich noch einmal ein halbes Jahrhundert, bis der kulturvergleichende 
Ansatz die Sozial- und Geisteswissenschaften nach dem Ende des Zweiten Weltkriegs nachhaltig zu beeinflussen begann. Dabei beschränkten sich die meisten Studien bis in die achtziger Jahre des zwanzigsten Jahrhunderts auf die Gegenüberstellung von zwei bzw. einigen wenigen Ländern, zu denen die Forscher mehr oder weniger zufällig Zugang hatten. Eine Ausnahme im Feld der Kommunikations- und Medienwissenschaft bildet die von der UNESCO in Auftrag gegebene Foreign Images-Studie, die zunächst in 29 Ländern durchgeführt und später in 38 Ländern repliziert wurde (vgl. SrebernyMohammadi, Nordenstreng \& Stevenson 1984; Wu 2000).

Strittig ist freilich noch immer die Frage der Definition von komparativer Forschung. Zwei extreme Positionen lassen sich im aktuellen Diskurs ausmachen: Traditionelle Definitionen beschränken komparative Forschung auf den Vergleich von zwei oder mehr territorialstaatlich abgrenzbaren Populationen (vgl. z. B. Edelstein 1982: 14). Allerdings sind Nationalstaaten häufig alles andere als abgeschlossene und homogene Einheiten; sie setzen sich vielmehr aus multiplen Kulturen zusammen (vgl. Livingstone 2003: 479). Andererseits hat die Wahl von Nationen als Vergleichseinheiten einen praktischen Vorteil: Sie verfügen über klar definierte Grenzen und sind, weil sie oftmals als die einzig verfügbaren Vergleichseinheiten gelten, „besser als gar nichts“ (Hofstede 2001: 73).

Demgegenüber vertritt eine Reihe von Autoren die radikale These, dass im Prinzip alle sozialwissenschaftliche Forschung einem komparativen Ansatz folgt und die Verwendung des Begriffs „komparative Forschung“ somit nur unnötige Redundanz erzeugt (vgl. z. B. Beniger 1992: 35f.). Für letzteres Argument spricht jedenfalls der Umstand, dass jede Einordnung von neuen Forschungsergebnissen tatsächlich nur über den Vergleich mit dem bestehenden Wissensbestand funktioniert. Andererseits existiert eine Reihe von methodologischen Herausforderungen, die offenbar nicht für jedes Forschungsdesign gleichermaßen relevant sind und die insbesondere im Hinblick auf den vergleichenden Ansatz spezifische Probleme aufwerfen. Hierzu gehören Faktoren wie die unterschiedliche Akzeptanz von Erhebungsmethoden in verschiedenen Kulturen oder die Art und Weise der Übersetzung von Erhebungsinstrumenten, die weiter unten ausführlich diskutiert werden.

Unstrittig ist, dass ein Vergleich räumlich (spatial, geografisch) und zeitlich (temporal, historisch) erfolgen kann, wobei freilich auch eine Kombination beider Varianten möglich ist. ${ }^{2}$ Die definitorische Sollbruchstelle bildet jedoch die Bestimmung der sozialen Vergleichseinheiten. Ist der Vergleich von Journalisten und Journalistinnen schon komparative Forschung? Wo lässt sich etwa die Kontrastierung von Fernsehgewohnheiten in Ost- und Westdeutschland verorten? Rechtfertigt die Gegenüberstellung des Internet-Nutzerverhaltens von Erwachsenen und Jugendlichen das Label „kulturvergleichend"?

Eine Klärung dieser Fragen ist zentral für die komparative Forschung, aber auch für unsere Meta-Erhebung, denn die Definition entscheidet über den Forschungsgegenstand. Vier Punkte können dazu bei komparativer Forschung zugrunde gelegt werden. Es muss erstens die Absicht des Vergleichs im Vordergrund stehen und im Forschungsdesign angelegt sein. Zweitens müssen die sozialen Vergleichseinheiten klar definiert sein und sich im Hinblick auf mindestens ein kulturelles Merkmal (nationale Herkunft, Geschlecht, Kohorte etc.) unterscheiden. Die Vergleichspopulationen müssen drittens hinsichtlich mindestens einer funktional äquivalenten Dimension verglichen werden.

2 Die zeitliche Komponente des Vergleichs soll im Folgenden ausgeblendet bleiben, da sie für diesen Beitrag keine Rolle spielt. 
Darüber hinaus heißt „Vergleichen“ viertens, dass die Vergleichseinheiten explizit aufeinander bezogen werden müssen.

Auf dieser Basis lässt sich ein vorläufiges Klassifikationsraster bestimmen, in das Beiträge zur komparativen Forschung eingeordnet werden können: Bei genuinen Vergleichen treffen alle vier oben genannten Punkte uneingeschränkt zu. Dahingegen handelt es sich bei kontextualisierenden Studien im Wesentlichen um monokulturelle Untersuchungen, in denen der Vergleich nur eine relativ untergeordnete Rolle spielt und zumeist der Kontextualisierung der Befunde dient. Überblicke zeichnen sich dadurch aus, dass die sozialen Vergleichseinheiten jeweils separat voneinander dargestellt und kaum aufeinander bezogen werden.

\subsection{Methodologische Standards}

In einer Analyse von Beiträgen aus sechs internationalen Fachzeitschriften, gestreckt über einen Zeitraum von 1970 bis 1997, haben Chang et al. (2001: 419ff.) der komparativen Kommunikationsforschung ein schlechtes Zeugnis ausgestellt. ${ }^{3}$ Beinahe zwei Drittel aller untersuchten Artikel waren atheoretisch, in den meisten Fällen wurde die Auswahl der Vergleichseinheiten nicht begründet. Darüber hinaus wurden häufig Populationen miteinander verglichen, die streng genommen eigentlich nicht vergleichbar sind. Chang et al. leiten daraus erhebliche Zweifel an der Gültigkeit der in den jeweiligen Beiträgen referierten Befunde und Schlussfolgerungen ab.

Mittlerweile hat sich das Fach jedoch weiterentwickelt. Im Hinblick auf die spezifischen Anforderungen an komparative Forschung hat auch in der Kommunikations- und Medienwissenschaft in den vergangenen zehn Jahren eine Diskussion grundlegender methodologischer Standards eingesetzt. Im Zentrum stehen dabei vor allem die theoretischen und philosophischen Grundlagen komparativer Forschung (vgl. Gudykunst \& Nishida 2000; Jensen 1998), die Problematik der funktionalen Äquivalenz (vgl. Wirth \& Kolb 2003), die Frage der Auswahl der Vergleichseinheiten (vgl. Ebbinghaus 2005) sowie allgemeine Problemstellungen und Lösungsansätze (vgl. Chang et al. 2001; Johnson \& Tuttle 2000; Livingstone 2003). Mit Blick auf den aktuellen methodologischen Diskussionsstand lassen sich folgende Qualitätsanforderungen an komparative Forschung qualifizieren:

1. Das komparative Vorgehen sollte begründet und die Zielsetzung des Vergleichs explizit gemacht werden (vgl. Chang et al. 2001: 420; Gurevitch \& Blumler 2003: 380). Die Frage, welchen besonderen Nutzen sich die beteiligten Forscher von der vergleichenden Methode versprechen, muss beantwortet werden. Der Leser einer wissenschaftlichen Abhandlung sollte nachvollziehen können, warum ein komparatives Vorgehen zwingend notwendig war (vgl. Scheuch 1990: 27). Angesichts des hohen Aufwandes für kulturvergleichende Forschungen - und hier insbesondere in Anbetracht der außerordentlichen methodologischen Anstrengungen - müssen sich Forscher stets fragen, ob die damit verbundene Aufwertung den Aufwand rechtfertigt: „Unless one has a good reason why research should be cross-national, it generally isn't worth the effort of making it cross-national.“ (Kohn 1989a: 97; kursiv im Original)

2. Befunde aus kulturvergleichender Forschung lassen sich ohne theoretische Anbindung oft kaum sinnvoll interpretieren. Die Forschung sollte daher in einer theoretischen

3 Die Zeitschriften waren im Einzelnen: Critical Studies in Mass Communication, European Journal of Communication, Gazette, Journal of Broadcasting and Electronic Media, Journal of Communication und Journalism \& Mass Communication Quarterly. 
Perspektive verankert sein, die sowohl die Sicht auf den Untersuchungsgegenstand als auch die vergleichende Untersuchungsanlage prägt (vgl. Gurevitch \& Blumler 2003: 380). Andernfalls wird es der komparativen Forschung kaum gelingen, über oberflächliche Beobachtungen und banale Schlussfolgerungen hinauszukommen (vgl. Chang et al. 2001: 420; Wirth \& Kolb 2003: 111). Auf einer allgemeinen Ebene lassen sich im Hinblick auf Theoriesättigung fünf grundsätzliche Typen unterscheiden: atheoretische, theoriebezogene, theoriegeleitete, theoriebildende sowie theoriegeleitete und theoriebildende Forschungen. Im Unterschied zur theoriebezogenen Forschung, die eine zumeist lose Anbindung an vorhandene Theorien und Modelle sucht, lassen sich theoriegeleitete Untersuchungen bei der Definition der zentralen Konzepte und Interpretation der Befunde von einer theoretischen Perspektive dezidiert anleiten. Eine solche theoretische Anleitung liegt auch bei der theoriegeleiteten und theoriebildenden Untersuchung vor, die darüber hinaus aber explizit an der (Weiter-)entwicklung von Theorie interessiert ist.

3. Die Einbindung in eine theoretische Perspektive ist nicht zuletzt auch unerlässlich für die systematische Auswabl der sozialen Vergleichseinheiten. Die Selektion der kulturellen Populationen hat einen irreversiblen Einfluss auf die Forschungsresultate, und sie bestimmt auch darüber, welche Ziele ein komparativer Ansatz erreichen bzw. nicht erreichen kann. Während etwa der Vergleich von ähnlichen Ländern („Most Similar Systems Design“) besser dazu geeignet ist, die Ursachen kultureller Varianz zu erklären, besteht das Potenzial des Vergleiches von sehr verschiedenen Ländern („Most Different Systems Design“) in der Untersuchung der Geltungskraft von vermeintlich universellen Aussagen (vgl. Przeworski \& Teune 1970: 31ff.). Welches Modell für den Vergleich herangezogen wurde, sollte expliziert und begründet werden, das Gleiche gilt für die Auswahl der sozialen Vergleichseinheiten (vgl. Ebbinghaus 2005; Livingstone 2003: 492; Teune 1990: 45). Wirth und Kolb (2003: 117) schlagen, der komparativen Logik folgend, für die theoriegeleitete und systematische Auswahl der Kulturen bzw. Länder eine Anlehnung an quasi-experimentelle Designs vor.

4. Das vielleicht wichtigste Qualitätskriterium komparativer Forschung ist die Wahrung bzw. Herstellung von Äquivalenz im Hinblick auf die verwendeten Konstrukte, Forschungsmethoden, administrativen Prozeduren, Forschungsinstrumente, Populationen und - falls zutreffend - Stichproben (vgl. Wirth \& Kolb 2003: 120ff.; van de Vijver \& Leung 1997: 8ff.). Konstruktäquivalenz impliziert, dass die zentralen Konstrukte (z. B. „Autonomie”) in allen teilnehmenden Kulturen übereinstimmend verstanden werden. Die Äquivalenz von Konstrukten sollte bereits während der Untersuchungsplanung und Konstruktion der Forschungsinstrumente (evtl. über international zusammengesetzte Forscherteams) berücksichtigt werden, sie kann aber auch nachträglich über statistische Verfahren bestimmt werden (z. B. mittels Reliabilitätsanalysen und Faktorstrukturvergleichen). Methoden- und Administrationsäquivalenz bezeichnet die Übereinstimmung hinsichtlich von Methoden und Durchführung (Administration) der Datenerhebung sowie die Kongruenz der Erhebungszeiträume. Instrumentenäquivalenz reicht über die inhaltliche Übereinstimmung der Forschungsinstrumente hinaus und betrifft auch ihre sprachliche Ausgestaltung (z. B. die Verbalisierung von Items einer Skala). Die Äquivalenz von Populationen und Stichproben schließlich bezieht sich auf die Vergleichbarkeit von Grundgesamtheiten und Methoden der Stichprobenziehung bzw. Verfahren der Fallauswahl. Für alle Formen von Äquivalenz gilt grundsätzlich: Sie kann niemals vorausgesetzt, sondern sollte vielmehr hergestellt werden (van de Vijver \& Leung 1997: 144). 
5. Die Frage, ob und wie Äquivalenz gesichert bzw. wie mit mangelnder Äquivalenz verfahren wird, ist essenziell für die Einschätzung und Einordnung von Ergebnissen komparativer Forschung. Deshalb ist eine möglichst vollkommene Transparenz über das methodische Vorgehen notwendig, auch bei der allerorten eingeforderten Kürze wissenschaftlicher Zeitschriftenbeiträge. Ohne diese Informationen können Außenstehende die Gültigkeit und Zuverlässigkeit der Ergebnisse nicht beurteilen. Die Publikation von komparativen Befunden ist für sich genommen weitgehend wertlos, wenn wesentliche methodologische Informationen fehlen.

Mit den Grundlagen komparativer Forschung sowie den methodologischen Standards sind die Rahmenbedingungen abgesteckt, innerhalb derer vergleichende Forschung arbeiten sollte. Bevor wir Ergebnisse dazu präsentieren, inwieweit diese Anforderungen erfüllt werden, soll kurz unser methodisches Vorgehen erläutert werden.

\subsection{Methodisches Vorgehen}

Unsere grundlegenden Fragestellungen lauteten: Wie lässt sich der Zustand der deutschen vergleichenden Kommunikationsforschung beschreiben? Werden die grundlegenden methodischen Anforderungen komparativer Forschung erfüllt? Dazu wurden mit der vorliegenden Studie kulturvergleichende Beiträge in deutschen kommunikationswissenschaftlichen Fachzeitschriften einer Meta-Analyse unterzogen. Als Analysekriterien dienten die oben vorgestellten Qualitätsstandards. Auf der inhaltlichen Ebene standen Fragen im Vordergrund, die auf die Untersuchungsgegenstände und theoretische Einbettung der analysierten Beiträge abzielten: Was sind die Themen der komparativen Forschung, welche wissenschaftlichen Fragestellungen wurden untersucht? Welche kulturellen Räume stehen im Vordergrund? Auf welche Theorien haben sich die Autoren bezogen? Welche Rolle hat die Theorie in den jeweiligen Untersuchungen gespielt? Wie hat sich die komparative Forschung im deutschsprachigen Raum im Zeitverlauf entwickelt?

Auf methodischer Ebene wurde untersucht, ob und wie die deutsche komparative Medien- und Kommunikationswissenschaft den besonderen methodologischen Anforderungen von kulturvergleichender Forschung gerecht wird. Ist es den Autoren gelungen, funktionale Äquivalenz im Hinblick auf die verwendeten Konstrukte, Forschungsmethoden, Administration, Forschungsinstrumente, Untersuchungszeiträume, Populationen und Stichproben sicherzustellen? Fanden kulturelle Spezifika bei Konzeptspezifikation, Wahl der Erhebungsmethoden, Konstruktion der Forschungsinstrumente, Bestimmung der Grundgesamtheiten und Stichprobenziehung bzw. Fallauswahl Berücksichtigung? Im Einzelnen wurde gefragt: Haben die Autoren der untersuchten Beiträge ihr komparatives Vorgehen und die Länderauswahl hinreichend begründet? Welche Begründungen wurden geliefert? Welche methodischen Verfahren (Datenerhebung, Stichprobenziehung bzw. Fallauswahl etc.) dominieren die kulturvergleichende Forschung?

$\mathrm{Da}$ eine abschließende Definition von komparativer Forschung noch nicht vorliegt, konzentriert sich die vorliegende Untersuchung auf die Analyse von ländervergleichenden Studien als speziellem Anwendungsfall der komparativen Forschung und hier insbesondere der kulturvergleichenden Forschung.

Fachzeitschriften bilden - stärker als Monografien und Sammelbände - als wissenschaftliche Leitmedien die Arena, in der fachliche Debatten ausgetragen, theoretische und methodologische Paradigmen durchgesetzt sowie wissenschaftliche Qualitätskriterien verhandelt werden. Die Grundlage der Inhaltsanalyse bilden alle entsprechen- 
den Artikel in den kommunikations- und medienwissenschaftlichen Fachzeitschriften. Untersucht wurden alle Ausgaben der Zeitschriften Medien E Kommunikationswissenschaft (früher: Rundfunk und Fernsehen, 1948-2005), Publizistik (1956-2005) und Media Perspektiven (1970-2005). Die Fachzeitschriften haben eine zentrale Bedeutung für die Fachwissenschaft, aber sie enthalten natürlich nicht alle Beiträge zur komparativen Forschung, die darüber hinaus in Monografien und Sammelbänden gebündelt sind. Dies ist bei der Bewertung der Ergebnisse zu berücksichtigen.

Um aus den hochgerechnet etwa 3.000 Beiträgen die Grundgesamtheit zu ermitteln, wurde ein Kriterienkatalog zur schnellen Einordnung herangezogen: Der komparative Charakter sollte demnach aus dem Titel oder Zwischenüberschriften, Abstracts, Tabellen oder grafischen Darstellungen deutlich werden. Explizit ausgeschlossen wurden Vergleiche, die z. B. auf einer Gegenüberstellung von ethnischen, genderspezifischen oder sprachraumbezogenen Kulturen beruhen, sowie Vergleiche innerhalb eines Staates (zum Beispiel Ost- und Westdeutschland). Es gelangten nur wissenschaftliche Aufsätze und Forschungsberichte in die Auswahl, Rezensionen wurden ausgeschlossen. Auf diese Weise ergab sich eine Zahl von 142 Beiträgen, die nach einer vollständigen Durchsicht schließlich auf 61 reduziert werden musste. Viele Beiträge schienen beim Anlegen der Screening-Kriterien (Titel, Zwischenüberschriften, Abstracts, Tabellen, grafische Darstellungen) einen Vergleich zu versprechen, die Texte haben diese Erwartungen jedoch nicht erfüllt. Von den 61 Artikeln entfielen 14 auf Medien E Kommunikationswissenschaft bzw. Rundfunk und Fernsehen, 15 Artikel auf die Publizistik und 32 Artikel auf Media Perspektiven.

Alle Beiträge wurden von den Autoren dieses Beitrags zunächst jeweils individuell und voneinander unabhängig kodiert, danach wurden die Codesheets auf Abweichungen geprüft. In späteren Arbeitssitzungen wurden die abweichenden Kodierungen ausführlich besprochen, dabei konnte in allen Fällen eine gemeinsame Entscheidung getroffen werden. Aufgrund der Komplexität des Materials war ein solches zeit- und arbeitsaufwendiges Verfahren unumgänglich. Die Alternative hätte in einer höheren Komplexitätsreduktion - und damit einem entsprechenden Informationsverlust - bestanden.

Im Folgenden werden die zentralen Befunde der Studie vorgestellt. Wir beschränken uns dabei auf die Auswertung der Beiträge, die in die Kategorien „Vergleich“ (39) und „Kontextualisierung“" (13) fallen $(\mathrm{N}=52)$. Die neun von uns als „Überblicksbeiträge“ klassifizierten Texte wurden ausgeschlossen, da sie aufgrund des mangelnden Bezugs zwischen den jeweiligen Untersuchungseinheiten keinen Vergleich im engeren Sinne bilden.

\section{Ergebnisse der Inhaltsanalyse}

\subsection{Entwicklung und Inbalte der komparativen Forschung}

Die Diagnose, wonach die komparative Medien- und Kommunikationsforschung in Deutschland nur ein Schattendasein führt (vgl. Kleinsteuber 2003: 78), trifft - nach den vorliegenden Daten zu urteilen - insbesondere auf die Zeit vor $1970 \mathrm{zu}$. Ab Mitte der 1970er Jahre erlebte die ländervergleichende Forschung einen beachtlichen Aufschwung, möglicherweise genährt durch die in dieser Zeit aufkommende Diskussion um eine neue Weltinformationsordnung. In diese Kategorie fallen u. a. die Beiträge von Breede (1985) und Heyn (1985) in den Media Perspektiven sowie von Wilke (1986) in der Publizistik.

Die Konjunktur der komparativen Kommunikationsforschung setzte jedoch erst 
in den 1990er Jahren ein: Über 40 Prozent der identifizierten Artikel entfallen allein auf den letzten untersuchten Zehnjahreszeitraum (1996-2005). Neben den eingangs geschilderten erleichterten Bedingungen für kulturvergleichende Forschung hat sich dabei insbesondere Europa zu einem „Forschungslaboratorium“ entwickelt: Einerseits durch die Herausbildung europäischer Strukturen wie z. B. Arte (1992) und 3sat (seit 1993 als Vollprogramm), die sich als kulturübergreifendes Forschungsobjekt eignen (z. B. in Bezug auf die Publikumsakzeptanz, vgl. Schroeder 1996). Darüber hinaus konnten Forscher nunmehr auch auf länderübergreifende Forschungsinstrumente zurückgreifen, u. a. auf den SES ASTRA Satellite Monitor (vgl. Keinath 2000). Transnationale Forschernetzwerke wie das Eurofiction-Projekt generieren zudem eine erhebliche Menge von Publikationen (vgl. Hallenberger 2003; 2005). Insgesamt ist der Aufwärtstrend der komparativen Forschung innerhalb der deutschen Wissenschaftskultur als robust zu kennzeichnen.

Von dieser Entwicklung am meisten profitiert haben zeitlich gesehen die Kommunikatorforschung und der Systemvergleich, auch die Produktforschung hat sich quantitativ verbessert. Von den Beiträgen, die sich eindeutig zuordnen lassen (44 von 52), entfallen immer noch allein über zwei Drittel auf die Produktforschung und den Systemvergleich. Die in der Kommunikationswissenschaft ansonsten dominante Rezipienten- und Wirkungsforschung macht hingegen zusammen nur reichlich ein Fünftel der Studien aus. Allerdings sind die einzelnen Kategorien nur schwer voneinander zu trennen. So lässt sich nur ein einziger Artikel eindeutig der Organisationsforschung zuordnen, eine größere Zahl an Aufsätzen ist dagegen zwischen Organisationsforschung und Systemvergleich angesiedelt, beispielsweise wenn die Radiolandschaft in verschiedenen Ländern untersucht wird. Die Dominanz der Produktforschung und des Systemvergleichs in der ländervergleichenden Forschung ist zweifellos dem Umstand geschuldet, dass komparative Inhaltsanalysen mit einem relativ geringen Ressourceneinsatz zu bewältigen sind. Für Systemvergleiche werden häufig aggregierte Daten aus sekundären Quellen oder öffentlichen (UNESCO, Freedom House etc.) bzw. kommerziellen Datenbanken (z. B. World Advertising Trends) herangezogen. Die Rezipienten- und Wirkungsforschung hingegen erfordert zum Teil erhebliche Forschungsmittel - ein Problem, das sich bei komparativer Forschung potenziert.

Im Hinblick auf die kommunikationswissenschaftlichen Untersuchungsgegenstände dominiert die Analyse von Informationsangeboten und politischer Kommunikation. Fast zwei Fünftel der untersuchten Beiträge beschäftigen sich explizit mit Journalismus, d. h. mit Nachrichtensendungen, Nachrichtenfaktoren, Auslandsberichterstattung, internationalen Nachrichtenflüssen, Wahlberichterstattung, Journalisten, Journalistikstudenten, der öffentlichen Meinung usw. Die Unterhaltungsproduktion steht - im Gegensatz zu ihrer zunehmenden Bedeutung im Programmangebot - eher selten im Mittelpunkt, und dann zumeist aus medienökonomischer Sicht und weniger in Bezug auf die Inhalte. Neben Untersuchungen zu Finanzierungs- und Kostenaspekten, die sich in der Schnittmenge von Organisationsforschung und Medienökonomie bewegen, spielt auch die wissenschaftliche Begleitung von technologischen Entwicklungen eine herausragende Rolle, insbesondere die Analyse des Zugangs zu und der Nutzung von neuen und digitalen Medienangeboten.

Die Verteilung der Länder, die für einen internationalen Vergleich herangezogen werden, bildet eine interessante Parallele zum Eurozentrismus der deutschen Auslandsberichterstattung, der sich in der Nachrichtengeografie (vgl. Schulz 1983) sowie der Streuung von Korrespondentenstandorten über den Globus (vgl. Junghanns \& Hanitzsch 2006) niederschlägt (vgl. Tabelle 1). Insbesondere Großbritannien und Frankreich 
sind häufig Gegenstand der komparativen Forschung, ebenso wie Österreich und die Schweiz, was mit Sicherheit auf die sprachliche Nähe zurückzuführen ist. So ist der Vergleich von Deutschland, Österreich und der (deutschsprachigen) Schweiz eine beliebte Kombination: Sie kam unter den untersuchten Beiträgen insgesamt sieben Mal vor, zwei weitere Beiträge beruhten auf einem deutsch-deutschen Vergleich (BRD und DDR).

Tabelle 1: Die zwölf am häufigsten verglichenen Länder $(N=332)$

\begin{tabular}{lc|lc}
\hline Land & $\mathbf{n}$ & Land & $\mathbf{n}$ \\
\hline BRD & 46 & USA & 16 \\
Großbritannien & 26 & Belgien & 13 \\
Frankreich & 22 & Spanien & 12 \\
Österreich & 20 & Dänemark & 11 \\
Italien & 19 & Niederlande & 11 \\
Schweiz & 17 & Schweden & 11 \\
\hline
\end{tabular}

Im Gegensatz zu ihrer zentralen Stellung im internationalen Wissenschaftsdiskurs rangieren die USA in der Rangliste der Vergleichsländer auf Platz sechs - wenn man Deutschland an dieser Stelle vernachlässigt. Wenig überrascht hat hingegen der Umstand, dass ganze Kontinente von der deutschen vergleichenden Kommunikationsforschung fast vollständig ausgeblendet wurden. Von den insgesamt 332 Ländernennungen ${ }^{4}$ entfielen nur fünf auf Afrika (Ägypten, Ghana, Nigeria, Tansania, Südafrika) und insgesamt vier auf Asien ohne Japan (Indien, Israel, Malaysia, Türkei).

Vieles spricht dafür, dass der europäische Raum für komparativ arbeitende Wissenschaftler am attraktivsten scheint. Dies mag insbesondere pragmatische Gründe haben: So haben die mittlerweile sieben europäischen Forschungsrahmenprogramme erhebliche Anreize für transnationale Forscherteams gesetzt, während die geografischen Distanzen in Europa überschaubar sind. Darüber hinaus bietet sich Europa aufgrund seiner kulturellen Vielfalt als internationales „Forschungslaboratorium“ geradezu an, da es einerseits kulturübergreifend zu beobachtende Forschungsobjekte generiert (z. B. „europäische Öffentlichkeit“, EU-Wahlberichterstattung, European Broadcasting Union) und eine breite Palette von potenziell interessanten Analyseeinheiten bereitstellt (Nationen, Sprachräume etc.).

\subsection{Begründungen komparativer Forschung}

Nach dieser Übersicht über die Inhalte und zeitliche Entwicklung der publizierten komparativen Forschung in deutschen Fachzeitschriften können wir nun damit beginnen, die bereits eingeführten theoretischen und methodologischen Qualitätsstandards anzulegen. Die erste Qualitätsanforderung, die wir in diesem Zusammenhang formuliert haben, richtet sich an die Begründung des komparativen Forschungsansatzes. Im Zentrum steht hierbei die Frage, ob die zwingende Notwendigkeit des komparativen Vorgehens nachvollziehbar begründet wurde. Unsere Befunde hierzu geben Grund zu Ernüchterung: In 34 der 52 untersuchten Arbeiten - das heißt in 65 Prozent - fand sich keinerlei Begründung für das komparative Vorgehen. Im Zeitverlauf ist zwar ein positiver Trend zu verzeichnen, der Anteil der Studien ohne explizite Begründung lag allerdings im

4 Die Summe aller Häufigkeiten des Vorkommens von Ländern. 
letzten untersuchten Zehnjahreszeitraum (1996-2005) immer noch bei 48 Prozent. Da für viele ländervergleichende Forschungspublikationen keine entsprechenden Begründungen mitgeliefert wurden, bleibt auch unklar, welchen konkreten Mehrwert sich die Autoren vom Kulturvergleich versprochen haben. Die Bilanz lautet an dieser Stelle allenfalls: „Gut, dass wir verglichen haben“" (Esser 2003).

Und selbst wenn die komparative Forschungsanlage explizit begründet wurde, so sagt dies noch nicht viel über die inhaltliche Qualität der Begründung aus. Die häufigsten Begründungen sind Verweise auf Forschungsdefizite (Tenor: „Hierfür liegen noch keine ländervergleichenden Studien vor.") sowie die Suche nach länderspezifischen Unterschieden. Schon der erste Fall macht deutlich, dass nicht alle - vielmehr tatsächlich nur die wenigsten - Begründungen auch zwingend nachvollziehbar waren. Eine gelungene Begründung hat z. B. Donsbach (1990: 408) in seinem Beitrag über JournalistikStudenten im internationalen Vergleich vorgelegt:

„In den Sozialwissenschaften setzt sich immer mehr die Erkenntnis durch, daß vergleichende Studien in vielen Fällen besser als Einzelanalysen in der Lage sind, soziale oder politische Merkmale einer Gesellschaft zu beschreiben. Dies gilt insbesondere für Forschungen über Normen, Werte und Einstellungen der Bevölkerung oder bestimmter Bevölkerungsteile. Während Einzelanalysen den Forscher im Ungewissen darüber lassen, wie bestimmte Häufigkeitsverteilungen, z. B. in den Antworten auf Fragen zu sozialen Normen, zu bewerten sind, ermöglichen Vergleichsdaten zu anderen Gruppen sehr rasch ein Urteil darüber, ob ein bestimmter Wert eine soziale Normalität oder eine Anomalie darstellt.“

\subsection{Theoretische Anbindung}

Die Notwendigkeit einer theoretischen Anbindung war das zweite Qualitätskriterium für komparative Forschung. Van de Vijver und Leung (1997: 20) sowie Wirth und Kolb (2003: 109) sprechen in diesem Zusammenhang von Theoriesättigung. Unsere Befunde deuten auch hier auf einen erheblichen Nachholbedarf für die vergleichende Forschung in Deutschland hin: In insgesamt 37 von 52 untersuchten Beiträgen (71 Prozent) wurden keinerlei Theorien zugrunde gelegt (vgl. Tabelle 2). In dieser Hinsicht stimmen unsere Ergebnisse mit der internationalen Studie von Chang et al. (2001: 422) überein. Zwar reduziert sich dieser Anteil im letzten Zehnjahreszeitraum (1996-2005) auf 57 Prozent, das heißt jedoch auch, dass immer noch mehr als die Hälfte der in deutschen kommunikationswissenschaftlichen Fachzeitschriften publizierten komparativen Beiträge atheoretisch arbeiten.

Insgesamt neun Beiträge klassifizierten wir als „theoriebezogen“, d. h. die Texte stellen einen expliziten Bezug zu theoretischen Ansätzen her. Weitere fünf Artikel waren theoriegeleitet in dem Sinne, dass sie nicht nur mehr oder minder lose Bezüge herstellen, sondern die Forschungsanlage sowie Interpretation der Befunde theoretisch fundieren. Ein Beitrag zeichnete sich dadurch aus, dass er einerseits theoriegeleitet war, andererseits aber auch einen Beitrag zur Theoriebildung (bzw. Theorie-Respezifikation) leistete. Die ursprünglich vorgesehene Kategorie „theoriebildend“ blieb leer. Die Palette der verwendeten theoretischen Perspektiven streut dabei ziemlich breit zwischen "klassischen“ kommunikationswissenschaftlichen Ansätzen, wie der Nachrichtenwerttheorie, Agenda-Setting und Medienimperalismus, sowie der Ästhetiktheorie und „Völkerpsychologie". 
Hanitzsch/Altmeppen · Komparative Forschung 1948-2005

Tabelle 2: Theoriebezug komparativer Forschung

\begin{tabular}{lcccc}
\hline & bis $\mathbf{1 9 6 5}$ & $\mathbf{1 9 6 6 - 1 9 8 5}$ & $\mathbf{1 9 8 6 - 2 0 0 5}$ & Gesamt \\
\hline atheoretisch & 2 & 17 & 18 & 37 \\
theoriebezogen & 1 & 1 & 7 & 9 \\
theoriegeleitet & 0 & 0 & 5 & 5 \\
theoriegeleitet \& theoriebildend & 0 & 0 & 1 & 1 \\
$\mathbf{N}$ & $\mathbf{3}$ & $\mathbf{1 8}$ & $\mathbf{3 1}$ & $\mathbf{5 2}$ \\
\hline
\end{tabular}

\subsection{Auswabl der Vergleichseinheiten}

Ein zentraler Aspekt der komparativen Forschung - und im speziellen Fall bei ländervergleichenden Studien - ist die Auswahl der Vergleichseinheiten, denn hier fällt bereits eine für die Interpretation der Ergebnisse wesentliche Vorentscheidung. Da eine Zufallsauswahl von Ländern, wie die Erfahrung zeigt, unrealistisch ist, kann eine systematische und im Idealfall theoriegeleitete Auswahl erheblich zum Wert einer komparativen Untersuchung beitragen (vgl. Ebbinghaus 2005). Über die quasi-experimentelle Auswahl der Vergleichseinheiten kann der Forscher nämlich eine Konstellation herbeiführen, die eine systematische Erklärung kultureller Varianz erlaubt. Dies kann z. B. über die Reduktion potenzieller Einflussvariablen im Most Similar Systems Design geschehen (vgl. Przeworski \& Teune 1970: 31ff.).

Die Befunde der vorliegenden Studie zeigen, dass dieses heuristische Potenzial in den untersuchten Beiträgen weitgehend nicht genutzt wurde. In nur 15 von 52 Aufsätzen wurde die Länderauswahl explizit begründet (29 Prozent), und ein positiver Trend ist auch im Zeitverlauf nicht erkennbar. Zum gleichen Ergebnis waren auch Chang et al. (2001: 424) in ihrer Inhaltsanalyse internationaler Fachzeitschriften gekommen. Die häufigsten Begründungen argumentierten mit der Ungleichheit $(n=4)$ bzw. Ähnlichkeit der Länder $(\mathrm{n}=3)$ sowie mit der Zugangsmöglichkeit ${ }^{5}(\mathrm{n}=2)$. Häufig waren Begründungen inhaltlicher Natur, wobei gerne auf die Verbreitung des Untersuchungsgegenstandes (z. B. sinngemäß: „Kabelfernsehen ist schon verbreitet“, vgl. Kellner 1981: 116) oder die Vorreiterrolle eines bestimmten Landes verwiesen wird (z. B. die USA bei der Entwicklung neuer Medien-Technologien, vgl. Buß 1980: 745). Eine in vielerlei Hinsicht gehaltvolle Begründung hat Wilke (1986: 60) in einem Beitrag zur Auslandsberichterstattung und zu internationalen Nachrichtenflüssen geliefert:

„Sich auf diese Länder zu stützen (und zunächst zu beschränken), dafür gibt es historische, sprachliche und praktische Gründe. Zeitungen sind hier seit dem 17. Jahrhundert (oder zumindest dem frühen 18. Jahrhundert) erschienen und materiell verfügbar. Diese Verfügbarkeit ist gegeben durch die Verfilmung historischer Zeitungsbestände (Mikrofilme), die in den vier Ländern heute schon weit gediehen ist. Sie ist eine unabdingbare Voraussetzung, um eine solch historisch komparative Studie überhaupt ausführen zu können. Hinzu kommt, dass Zeitungen aus Großbritannien, Frankreich und den Vereinigten Staaten von Amerika sprachlich zugänglich sind für die an der Inhaltsanalyse beteiligten Kodierer. Es handelt sich zudem um Länder von historisch-politischer Bedeutung, die während des Untersuchungszeitraums eine weitgehende geographisch-politische Identität bewahrten; indem das koloniale Amerika bzw. die späteren Vereinigten Staaten von Amerika einbezogen werden, läßt sich historisch vielleicht etwas von der Problematik eines „Entwicklungslandes“ einfangen, eine Problematik, die sich in heutiger Form sonst nur schwerlich rücktransponieren läßt.“

5 D. h. die Forscher hatten Zugang zu den verglichenen Ländern. Mehrfachcodierungen waren möglich. 


\section{5 Äquivalenz in der komparativen Forschung}

Im Hinblick auf die verwendeten Forschungsmethoden muss zunächst festgehalten werden, dass nur für 36 Beiträge (69 Prozent) methodische Angaben zumindest teilweise vorliegen. Dabei ist dieser Anteil in der untersuchten Zeitspanne kontinuierlich angestiegen, und zwar auf 81 Prozent im letzten untersuchten Zehnjahreszeitraum. Knapp die Hälfte aller Artikel $(n=24 ; 46$ Prozent) stützt sich auf Primärdaten, also eigene Erhebungen zum jeweiligen Erkenntnisgegenstand, die acht rein sekundäranalytischen Arbeiten machen 15 Prozent aus. Insgesamt sechs Beiträge wurden als Mischform eingestuft, weitere vier bedienten sich der Kontextanalyse, d. h. sie arbeiten mit aggregierten Daten aus wissenschaftlichen Quellen und transnationalen Datenarchiven und Datenbanken (UNESCO, International Telecommunication Union etc.). Zehn Artikel ließen die Herkunft der Daten vollkommen offen.

Bei den verwendeten Methoden dominieren quantitative Verfahren klar, sie wurden in 25 von insgesamt 32 Beiträgen, in denen auf die Erhebungsmethoden eindeutig geschlossen werden konnte, eingesetzt (78 Prozent). Nur zwei Studien bedienten sich qualitativer Erhebungsinstrumente, in fünf Fällen wurden qualitative und quantitative Methoden kombiniert. Die Dominanz quantitativer Forschungsdesigns ist dabei sicherlich auch dem Umstand geschuldet, dass sich das quasi-experimentelle Potenzial der komparativen Logik offenbar intuitiv am besten in ein quantitatives Methodenset übersetzen lässt. Daher ist auch die Einführungs- und Grundlagenliteratur im Feld der komparativen Forschung sehr stark von quantitativen Elementen geprägt (vgl. u. a. Landman 2000; van de Vijver \& Leung 1997).

Dort, wo etwas über die Erhebungsmethoden zu erfahren war, dominierten Befragungen $(n=29)$ vor Inhaltsanalysen $(n=15) .{ }^{6}$ Mündliche, schriftliche und telefonische Befragungen waren in der Beliebtheit dabei etwa gleichauf, auf die Online-Befragung wurde nur einmal zurückgegriffen. Aufwendigere Erhebungsverfahren wie Experiment oder Beobachtung waren in den untersuchten Beiträgen nicht vorhanden, wohl auch, weil die doppelte Komplexität von vergleichender Studie und anspruchsvoller Methode einen erheblich höheren Aufwand als bei traditionellen Instrumenten erfordert hätte. Der gleiche Grund ist sicherlich auch dafür verantwortlich, dass Querschnittstudien $(n=29)$ weit vor den Längsschnittuntersuchungen $(n=5)$ rangieren, wobei auch hier zu beachten ist, dass für insgesamt 18 Untersuchungen nicht einwandfrei festgestellt werden konnte, um welche der beiden Designs es sich handelte. Beispielhaft für Untersuchungen, in denen die Methodenäquivalenz nur bedingt berücksichtigt wurde, steht eine 22-Länder-Studie, die je nach Land mit einer telefonischen, mündlichen oder schriftlichen Befragung operierte. Unterschiedliche Grundgesamtheiten lassen sich u. a. dort erkennen, wo z. B. Länder auf der Basis eines jeweils sehr unterschiedlichen Gemischs von öffentlich-rechtlichen bzw. staatlichen Fernsehsendern einerseits und privat-kommerziellen TV-Programmen andererseits untersucht wurden.

\subsection{Transparenz und die Auseinandersetzung mit kulturellen Spezifika}

Die Erläuterung des methodischen Vorgehens bildet für die Darstellungen empirischer Ergebnisse einen unverzichtbaren Bestandteil, um Transparenz herzustellen und intersubjektive Nachprüfbarkeit zu ermöglichen. Das gilt in besonderem Maße für vergleichende Forschung, wobei das Problem weniger darin liegt, dass sich Forschungsdesigns

6 Mehrfachcodierungen waren aufgrund von Methodenkombinationen möglich. 
in den miteinander verglichenen Ländern unterscheiden können. Vielmehr geht es um die Frage, wie sich die Forscher diesen Problemen konkret gestellt haben. Tatsächlich aber war in insgesamt 20 untersuchten Studien keinerlei Hinweis - weder explizit noch implizit - auf die verwendeten Methoden zu finden. Nur 16 von 52 untersuchten Beiträgen (31 Prozent) haben Angaben zur Grundgesamtheit gemacht, und 17 von 51 Studien (33 Prozent) haben Auskunft über die verwendeten Auswahlverfahren gegeben. Wobei auch hier grob ein positiver Trend zu verzeichnen ist, wie im Vergleich der drei 20-Jahres-Zeiträume deutlich wird (vgl. Tabelle 3). In 13 Beiträgen wurde lediglich darauf hingewiesen, dass eine Auswahl stattgefunden hat. Insgesamt sind komplexere Auswahlverfahren eher selten, es dominieren Vollerhebungen, einfache und mehrstufige Zufallsstichproben und verschiedene Formen der bewussten Auswahl.

Die mangelhafte Transparenz im Hinblick auf das methodische Vorgehen hat die Konsequenz, dass jene Qualitätskriterien, die sich mit dem Begriff der Äquivalenz verbinden, oftmals gar nicht angelegt werden können. Für viele Studien wissen wir einfach nicht, ob die verwendeten Verfahren und Forschungsinstrumente auch tatsächlich äquivalent waren. Oftmals scheint der einfache Verweis darauf, dass die Feldforschung in allen beteiligten Ländern „prinzipiell vergleichbar“ durchgeführt wurde, den Autoren zu genügen. Tabelle 3 macht dies deutlich: Die am häufigsten codierte Kategorie mit Blick auf die Äquivalenz von Methoden, Instrumenten, Erhebungszeiträumen, Grundgesamtheiten und Auswahlverfahren war „unklar“. Insgesamt aber zeigt sich auch hier wieder ein positiver Trend über die untersuchten fast sechs Jahrzehnte.

\section{Tabelle 3: Aquivalenz von Methoden, Instrumenten, Erbebungszeiträumen, Grundgesamtheiten und Auswablverfabren}

\begin{tabular}{llcccc}
\hline & & bis 1965 & 1966-1985 & 1986-2005 & Gesamt \\
\hline Methoden & einheitlich & 0 & 7 & 16 & 23 \\
& nicht einheitlich & 0 & 2 & 2 & 4 \\
& unklar & 3 & 9 & 13 & 25 \\
\hline Instrumente & einheitlich & 1 & 4 & 8 & 13 \\
& nicht einheitlich & 0 & 1 & 1 & 2 \\
& unklar & 2 & 13 & 21 & 36 \\
\hline Erhebungs- & einheitlich & 0 & 6 & 13 & 19 \\
zeitraum & nicht einheitlich & 0 & 1 & 5 & 6 \\
& unklar & 3 & 11 & 13 & 27 \\
\hline Grundgesamt- & einheitlich & 0 & 3 & 10 & 13 \\
beit & nicht einheitlich & 0 & 0 & 2 & 2 \\
& unklar & 3 & 15 & 19 & 37 \\
\hline Auswabl- & einheitlich & 0 & 2 & 10 & 12 \\
verfabren & nicht einheitlich & 0 & 1 & 3 & 4 \\
& unklar & 3 & 15 & 17 & 35 \\
\hline
\end{tabular}

Eine hundertprozentige Äquivalenz in all diesen Bereichen ist in der sozialwissenschaftlichen Forschung praktisch jedoch kaum zu erreichen, das ist klar. Umso wichtiger ist dann die Auseinandersetzung mit der Frage, wie die Forscher mit der Problematik der kulturellen Spezifika (etwa von mündlichen Befragungen in verschiedenen kulturellen Kontexten), die mit kulturvergleichender Forschung stets einhergehen, im konkreten 
Anwendungsfall umgegangen sind. Die Ergebnisse zeigen allerdings, dass allein die Frage schon viel zu optimistisch formuliert war. Korrekterweise hätte die Frage lauten müssen, ob die Auseinandersetzung mit den kulturellen Spezifika überhaupt stattgefunden hat.

Tabelle 4 zeichnet in dieser Hinsicht ein klares Bild: Die in verschiedenen kulturellen Kontexten möglicherweise recht unterschiedlichen Verzerrungseffekte der verwendeten Datenerhebungsmethoden wurden in keinem der untersuchten Beiträge zur Sprache gebracht. So ist z. B. bekannt, dass die Befragten im Interview in jedem Fall versuchen werden, „angemessen“ auf die Befragungssituation zu reagieren (vgl. Kromrey 2000: 337), wobei die Definition dessen, was gemeinhin als angemessen gilt, jeweils kulturspezifisch ausfällt. Dies führt mit hoher Wahrscheinlichkeit zu unterschiedlich starken Befragteneffekten vor allem der sozialen Erwünschtheit, inhaltsunabhängigen Zustimmung (Akquieszenz), Non-Attitudes (inhaltliche Antwort trotz Meinungslosigkeit) und Verweigerung (vgl. für den asiatischen Raum: Hanitzsch 2004: 125ff.). Insbesondere Höflichkeitsnormen sind hier von besonderer Relevanz, was die in einigen Kulturen ausgeprägte Vermeidung von negativen bzw. Nein-Antworten erklärt (vgl. Dieckmann 1995: 377). Darüber hinaus spielt der gesellschaftliche Status von Interviewer und Befragtem in verschiedenen Kontexten eine unterschiedlich starke Rolle (vgl. Johnson \& Tuttle 2000: 474). Die inhaltliche Auseinandersetzung mit diesen Fragen wurde in den untersuchten Beiträgen nicht geführt, weshalb wir alle Artikel mit „unklar“ codieren mussten. Das muss nicht heißen, dass diese Auseinandersetzung überhaupt nicht stattfand. Dort, wo sie geführt wurde, haben es die Autoren möglicherweise nur versäumt, ihre Entscheidungen nachvollziehbar zu machen.

Auch im Hinblick auf die kulturellen Spezifika der Forschungsinstrumente waren wir zunächst davon ausgegangen, dass diese in vielen untersuchten Beiträgen zumindest diskutiert werden, auch wenn es den Autoren aus verschiedenen - z. B. forschungsökonomischen - Gründen nicht möglich war, diese Spezifika in ihren Untersuchungen hinreichend zu berücksichtigen. Aber auch hier mangelt es an Transparenz: In 39 von 43 Fällen (91 Prozent) hielten sich die Autoren in dieser Frage bedeckt. Ein Aufwärtstrend war jedoch im letzten untersuchten Zehnjahreszeitraum festzustellen. So wurden in insgesamt drei Beiträgen verschiedene Verfahren zur Sicherung der Äquivalenz der Untersuchungsinstrumente zur Anwendung gebracht. Mit gutem Beispiel voran geht Trepte (2004: 237), die bestimmte Variablen von der Analyse ausgeschlossen hat, die sich aufgrund von Homogenitäts- und Faktorenanalysen für einen Vergleich als untauglich erwiesen. In einem Fall wurden die Länderspezifika diskutiert, in der Untersuchung aber nicht berücksichtigt. Die in der Forschung bekannten Verfahren zur Äquivalenzsicherung - übersetzungsorientierte Ansätze (z. B. die Translation-Backtranslation-Prozedur), das cultural decentering und der Komitee-Ansatz (vgl. van de Vijver \& Leung 1997: 39ff.; Wirth \& Kolb 2003: 115) - fanden in keiner der untersuchten Arbeiten Erwähnung.

Ähnlich stellt sich die Lage bezüglich der Grundgesamtheiten und Auswahlverfahren dar. Diskutiert fand sich die Frage der Definition der Grundgesamtheiten in der Arbeit von Zöllner (2002: 233f.), der in seiner Akzeptanzstudie über Deutsche Welle TV feststellt, dass in Afghanistan während der Taliban-Herrschaft nur männliche Haushaltsvorstände befragt werden konnten und dass in vielen Entwicklungsländern der Anteil derer, die Zugang zu einem Fernsehgerät haben, im Vergleich zu westlichen Industriestaaten recht gering ist. Allerdings bleibt unklar, welche Auswirkungen dies auf die Interpretation der Ergebnisse hat und welche eventuellen Korrekturmaßnahmen ergriffen wurden. 
Tabelle 4: Berücksichtigung der kulturellen Spezifika im Hinblick auf Methoden, Instrumente, Grundgesamtheiten und Auswablverfabren ${ }^{7}$

\begin{tabular}{llrrrr}
\hline $\begin{array}{l}\text { Eine Auseinandersetzung } \\
\text { mit kulturellen Spezifika } \\
\text { fand statt bezüglich ... }\end{array}$ & bis 1965 & 1966-1985 & 1986-2005 & Gesamt \\
\hline Methoden & ja & 0 & 0 & 0 & \\
& unklar & 3 & 16 & 30 & 49 \\
\hline Instrumenten & ja & 0 & 0 & 4 & 4 \\
& unklar & 2 & 14 & 23 & 39 \\
\hline Grundgesamtheit & ja & 0 & 0 & 5 & 5 \\
& unklar & 3 & 18 & 26 & 47 \\
\hline Auswablverfabren & ja & 0 & 0 & 6 & 6 \\
& unklar & 3 & 17 & 21 & 41 \\
\hline
\end{tabular}

\section{Fazit und Ausblick}

Die vorliegende Untersuchung hat gezeigt, dass sich die Bedeutungszunahme der komparativen Forschung in der deutschen Medien- und Kommunikationswissenschaft quantitativ in wissenschaftlichen Fachzeitschriften niederschlägt. Im Hinblick auf die Beachtung grundlegender Qualitätsstandards empirischer Sozialforschung im Allgemeinen und komparativer Forschung im Besonderen offenbart sich jedoch ein größerer Nachholbedarf. Grundlegende Standards sozialwissenschaftlicher Forschung bleiben vielfach unberücksichtigt, womit die Autoren den Wert ihrer häufig aufwendig betriebenen Forschungsarbeit unterminieren.

Dennoch ist durchaus ein positiver Trend erkennbar, insbesondere für die im letzten untersuchten Zehnjahreszeitraum veröffentlichten Beiträge. Zudem lässt sich feststellen, dass die - in der Regel über Inhaltsanalysen realisierte - Produktforschung sowie Systemvergleiche zusammen mehr als zwei Drittel des gesamten Publikationsausstoßes im wachsenden Feld der komparativen Forschung ausmachen. Kulturvergleichende Inhaltsanalysen können zumeist ohne größeren Aufwand durchgeführt werden, und Systemvergleiche kommen in vielen Fällen sogar ohne eigene Datenerhebung aus, da sie aus einer Makro-Perspektive bereits publizierte Einzelbefunde aus verschiedenen kulturellen Kontexten mit öffentlich zugänglichen Daten auf Aggregatniveau analytisch zusammenführen können. Ein herausragendes Beispiel aus dem internationalen Raum ist die Studie „Comparing Media Systems: Three Models of Media and Politics“ von Hallin und Mancini (2004).

Darüber hinaus dominiert die quantitative Forschung das Feld ziemlich klar, als Erhebungsmethoden kamen - soweit bekannt - ausschließlich die Befragung und die Inhaltsanalyse mit zumeist strukturierten Instrumenten zum Einsatz. Angesichts der in den Einrichtungen der Wissenschaftsförderung vorherrschenden Tendenz zur Finanzierung von relativ „kurzen“ Forschungsprojekten (von zumeist bis zu drei Jahren)

7 Wie bei Tabelle 3 variiert $\mathrm{N}$ im Hinblick auf die verschiedenen untersuchten Merkmale. Einige Kriterien treffen nicht auf alle Beiträge zu. So ist etwa die Frage der sprachlichen Äquivalenz bei Inhaltsanalysen von deutschsprachigen Tageszeitungen in Deutschland, Österreich und der Schweiz nicht relevant. 
kann es auch kaum verwundern, dass die Zahl der Querschnittstudien die der Längsschnitte bei weitem übertrifft. Im Hinblick auf die Länderauswahl dominiert sehr stark der europäische Raum, die USA finden sich trotz ihrer dominanten Position in der kommunikationswissenschaftlichen Fach-Community nur auf einem überraschenden siebten Platz.

Die vorliegende Vollerhebung von Zeitschriftenaufsätzen in deutschen Fachzeitschriften hat allerdings auch zahlreiche Probleme deutlich gemacht. So liefern die meisten Studien keinerlei Begründung für das komparative Vorgehen und die Auswahl der Länder. Der Leser erfährt in vielen Fällen nichts über die Zielsetzung des Vergleichs und den besonderen Nutzen, der den zum Teil immensen methodischen Aufwand rechtfertigen würde. Zudem ist die komparative Forschung nur ungenügend in eine theoretische Perspektive eingebettet, der größte Teil der untersuchten Arbeiten ist atheoretischer $\mathrm{Na}$ tur. Zu wichtigen Qualitätsstandards fehlen oftmals hinreichende Angaben, insbesondere im Hinblick auf die verwendeten Erhebungsmethoden, Grundgesamtheiten und Verfahren der Fallauswahl (bzw. der Stichprobenziehung). Zwar ist auch hier im zeitlichen Vergleich eine positive Tendenz auszumachen, dennoch waren im letzten Drittel des Untersuchungszeitraums (also von 1986 bis 2005) bei fast zwei Dritteln der Beiträge keine Angaben zur Grundgesamtheit zu finden. Bei über der Hälfte der Beiträge fehlten sogar jegliche konkreten Angaben zur Auswahl der Fälle.

Unklar bleibt vor allem, inwieweit die kulturellen Spezifika der unterschiedlichen Länder - soweit dies von Relevanz war - in den jeweiligen Studien berücksichtigt wurden. Bezüglich der forschungsleitenden Konstrukte, Forschungsinstrumente, Grundgesamtheiten und Verfahren zur Auswahl der Untersuchungseinheiten war es in weit über 80 Prozent der Fälle unmöglich einzuschätzen, ob und wie sich die beteiligten Forscher diesem Problem gestellt haben. Hinsichtlich der kulturellen Spezifika von Erhebungsmethoden gab es in keiner der untersuchten Studien verwertbare Angaben. Damit ist auch das entscheidende Problem der deutschen komparativen Forschung beschrieben: Der Mangel an Transparenz und intersubjektiver Überprüfbarkeit macht es der wissenschaftlichen Leserschaft dieser Beiträge unmöglich, die Gültigkeit und Zuverlässigkeit der Ergebnisse hinreichend zu beurteilen.

Es lässt sich freilich berechtigterweise einwenden, dass viele der oben genannten Qualitätsstandards angesichts der objektiven Forschungsbedingungen oft nur begrenzt erreichbar sind. Vor allem der mit der Herstellung von funktionaler Äquivalenz verbundene Aufwand ist in vielen Fällen schlicht nicht finanzierbar. Gerade für diese Fälle aber ist eine vollständige Transparenz im Hinblick auf das methodische Vorgehen und den Umgang mit den spezifischen kulturellen Bedingungen essenziell. Es ist unbedingt anzuraten, dass bei zur Veröffentlichung eingereichten komparativen Beiträgen detaillierte methodische Informationen eingefordert werden und dass die Herausgeber von Fachzeitschriften ihren Autoren den hierfür notwendigen Raum zubilligen.

Bei der Interpretation der hier dargestellten Ergebnisse sollte allerdings berücksichtigt werden, dass nur ländervergleichende Studien in die Analyse eingegangen sind. Darüber hinaus wurden nur Beiträge in den drei führenden deutschen Fachzeitschriften untersucht - unberücksichtigt blieb damit, dass in der deutschsprachigen Wissenschaftskultur viele wichtige Arbeiten in Sammelbänden und Monografien publiziert werden. Eine Analyse von Fachzeitschriften kann also nur Anhaltspunkte für den Zustand der deutschen komparativen Kommunikations- und Medienforschung liefern.

Zudem können Publikationen allein nicht mit Sicherheit darauf schließen lassen, dass die Autoren der Beiträge die von uns dargestellten Qualitätskriterien missachtet haben. Wir wissen nur das, was die jeweiligen Forscher auch mitgeteilt haben. Ob und 
inwiefern etwa Erhebungsmethoden tatsächlich identisch waren, können wir für jene Beiträge, die hierzu keinerlei Angaben gemacht haben, nicht beurteilen. Dennoch zählt in der Wissenschaft allein das, was auch veröffentlicht - das heißt publiziert und auf Konferenzen vorgetragen - wurde. In einigen wenigen Artikeln wurde zumindest auf vorangegangene oder begleitende Veröffentlichungen verwiesen, die methodische Details enthalten. Eine Erweiterung der Analyse auch auf diese Publikationen würde das Bild der komparativen Forschung zweifellos vervollständigen, sie war im Rahmen der vorliegenden Untersuchung aber nicht möglich.

\section{Literatur}

Beniger, James R. (1992): Comparison, Yes, But - The Case of Technological and Cultural Change. In: Jay G. Blumler, Jack M. McLeod \& Karl Erik Rosengren (eds.): Comparatively Speaking: Communication and Culture across Space and Time. Newbury Park, CA: Sage, 35-50.

Blumler, Jay G., Jack M. McLeod \& Karl Erik Rosengren (1992): An Introduction to Comparative Communication Research. In: Jay G. Blumler, Jack M. McLeod \& Karl Erik Rosengren (eds.): Comparatively Speaking: Communication and Culture across Space and Time. Newbury Park, CA: Sage, 3-18.

Breede, Werner E. (1985): UNESCO-Studie '83: Internationaler Fluß von Fernsehprogrammen und Nachrichten. Ausgewählte Ergebnisse für die Bundesrepublik Deutschland und Westeuropa. In: Media Perspektiven 3: 214-223.

Buß, Michael (1980): Videorecorder statt Verkabelung? Die Nutzung von Videogeräten in der Bundesrepublik Deutschland und in den USA. In: Media Perspektiven 11: 745-750.

Chang, Tsan-Kuo with Pat Berg, Anthony Ying-Him Fung, Kent D. Kedl, Caterine A. Luther \& Janet Szuba (2001): Comparing Nations in Mass Communication Research, 1970-97: A Critical Assesment of How We Know What We Know. In: Gazette 63 (5): 415-434.

Dieckmann, Rainer (1995): Empirische Sozialforschung. Grundlagen, Methoden, Anwendungen. 4., durchges. Aufl. Reinbek: Rowohlt.

Donsbach, Wolfgang (1990): Journalistikstudenten im internationalen Vergleich. In: Publizistik 35 (4): 408-427.

Durkheim, Emil (1897/1973). Der Selbstmord. Neuwied: Luchterhand.

Ebbinghaus, Bernhard (2005): When Less is More: Selection Problems in Large-N and Small-N Cross-National Comparisons. In: International Sociology 20 (2): 133-152.

Edelstein, Alex S. (1982): Comparative Communication Research. Beverley Hills, CA: Sage.

Esser, Frank (2003): Gut, dass wir verglichen haben. Bilanz und Bedeutung der komparativen politischen Kommunikationsforschung. In: Frank Esser \& Barbara Pfetsch (Hrsg.): Politische Kommunikation im internationalen Vergleich. Grundlagen, Anwendungen, Perspektiven. Wiesbaden: Westdeutscher Verlag, 437-494.

Esser, Frank \& Barbara Pfetsch (Hrsg.) (2003): Politische Kommunikation im internationalen Vergleich. Grundlagen, Anwendungen, Perspektiven. Wiesbaden: Westdeutscher Verlag.

Gudykunst, William B. \& Tsukasa Nishida (2000): Theoretical Perspectives for Studying Intercultural Communication. In: Molefi Kete Asante \& William B. Gudykunst (eds.): Handbook of International and Intercultural Communication. Newbury Park, CA: Sage, 17-46.

Gurevitch, Michael \& Jay G. Blumler (2003): Der Stand der vergleichenden politischen Kommunikationsforschung: Ein eigenständiges Feld formiert sich. In: Frank Esser \& Barbara Pfetsch (Hrsg.): Politische Kommunikation im internationalen Vergleich. Grundlagen, Anwendungen, Perspektiven. Wiesbaden: Westdeutscher Verlag, 371-392.

Hallenberger, Gerd (2003): Eurofiction 2002: Trotz Krise überraschend stabiles Angebot. Erstausgestrahlte einheimische fiktionale Fernsehproduktionen in Deutschland. In: Media Perspektiven 11: 490-499.

Hallenberger, Gerd (2005): Eurofiction 2003: Deutlicher Angebotsrückgang. Erstausgestrahlte einheimische fiktionale Fernsehproduktionen in Deutschland: Angebotsstruktur und Nutzung. In: Media Perspektiven 1: 14-22. 
Hallin, Daniel C. \& Paolo Mancini (2004): Comparing Media Systems: Three Models of Media and Politics. New York: Cambridge University Press.

Hanitzsch, Thomas (2004): Journalismus in Indonesien. Akteure, Strukturen, Orientierungshorizonte, Journalismuskulturen. Wiesbaden: Deutscher Universitäts-Verlag.

Heyn, Jürgen (1985): Fernsehnachrichten im internationalen Vergleich. Auf bestem Wege zur Entpolitisierung? In: Media Perspektiven 12: 879-884.

Hofstede, Geert (2001): Culture`s Consequences. Second Edition: Comparing Values, Behaviors, Institutions and Organizations across Nations. Thousand Oaks: Sage.

Jensen, Klaus Bruhn (1998): Local Empiricism, Global Theory: Problems and Potentials of Comparative Research on News Reception. In: Communications, 23 (4): 427-445.

Johnson, J. David \& Frank Tuttle (2000): Problems in Intercultural Research. In: Molefi Kete Asante \& William B. Gudykunst (eds.): Handbook of International and Intercultural Communication. Newbury Park, CA, London \& New Delhi: Sage, 461-483.

Junghanns, Kathrin \& Thomas Hanitzsch (2006): Deutsche Auslandskorrespondenten im Profil. In: Medien \& Kommunikationswissenschaft 55 (3): 412-429.

Keinath, Annette (2000): Fernsehempfang und PC/Online-Ausstattung in Europa. Ergebnisse des SES/ASTRA Satellite Monitors 2000. In: Media Perspektiven 10: 451-457.

Kellner, Hella (1981): Wieviel und welches Fernsehen wollen und nutzen die Bürger? In: Media Perspektiven 2: 116-123.

Kleinsteuber, Hans J. (2003): Medien und Kommunikation im internationalen Vergleich: Konzepte, Methoden und Befunde. In: Frank Esser \& Barbara Pfetsch (Hrsg.): Politische Kommunikation im internationalen Vergleich. Grundlagen, Anwendungen, Perspektiven. Wiesbaden: Westdeutscher Verlag, 78-103.

Kohn, Melvin L. (1989a): Cross-National Research as an Analytic Strategy. In: Melvin L. Kohn (ed.): Cross-National Research in Sociology. Newbury Park, CA: Sage, 77-102.

Kohn, Melvin L. (ed.) (1989b): Cross-National Research in Sociology. Newbury Park, CA: Sage.

Kromrey, Helmut (2000): Empirische Sozialforschung. Modelle und Methoden der standardisierten Datenerhebung und Datenauswertung. 9., korr. Aufl. Opladen: Leske + Budrich.

Landman, Todd (2000): Issues and Methods in Comparative Politics: An Introduction. London \& New York: Routledge.

Livingstone, Sonia (2003): On the Challenges of Cross-National Comparative Media Research. In: European Journal of Communication, 18 (4): 477-500.

Peter, Jochen (2003): Konsonanz 30 Jahre später. Eine international vergleichende Studie zum Einfluss konsonanter Berichterstattung auf Meinungen zur europäischen Integration. In: Publizistik 48 (2): 190-208.

Øyen, Else (ed.) (1990): Comparative Methodology: Theory and Practice in International Social Research. London: Sage.

Przeworski, Adam \& Henry Teune (1970): The Logic of Comparative Inquiry. New York: Wiley.

Scheuch, Erwin K. (1990): The Development of Comparative Research: Towards Causal Explanations. In: Else Øyen (ed.): Comparative Methodology: Theory and Practice in International Social Research. London: Sage, 19-37.

Schroeder, Michael (1996): Arte: Kulturprogramm mit europäischem Auftrag. Positionierung, Image und Akzeptanz von Arte in Frankreich und Deutschland. In: Media Perspektiven 2: 93-104.

Schulz, Winfried (1983): Nachrichtengeographie. Untersuchungen über die Struktur der internationalen Berichterstattung. In: Manfred Rühl \& Heinz-Werner Stuiber (Hrsg.): Kommunikationspolitik in Forschung und Anwendung. Düsseldorf: UVK, 281-291.

Sreberny-Mohammadi, Annabelle, Kaarle Nordenstreng \& Robert L. Stevenson (1984): The world of the news study. In: Journal of Communication 34 (1): 134-38.

Teune, Henry (1990): Comparing Countries: Lessons Learned. In: Else Øyen (ed.): Comparative Methodology: Theory and Practice in International Social Research. London: Sage, 38-62.

Trepte, Sabine (2004): Soziale Identität und Medienwahl. Eine binationale Studie zum Einfluss der Gender-Identität und nationaler Identität auf die Selektion unterhaltender Medieninhalte. In: Medien \& Kommunikationswissenschaft 52 (2): 230-249. 
Tylor, Edward B. (1871): Primitive culture: researches into the development of mythology, philosophy, religion, art, and custom. London: J. Murray.

van de Vijver, Fons J. R. \& Kwok Leung (1997): Methods and Data Analysis for Cross-Cultural Research. Thousand Oaks, CA: Sage.

Wilke, Jürgen (1986): Auslandsberichterstattung und internationaler Nachrichtenfluß im Wandel. In: Publizistik (31) 1: 53-90.

Wirth, Werner \& Steffen Kolb (2003): Äquivalenz als Problem: Forschungsstrategien und Designs der komparativen Kommunikationswissenschaft. In: Frank Esser \& Barbara Pfetsch (Hrsg.): Politische Kommunikation im internationalen Vergleich. Grundlagen, Anwendungen, Perspektiven. Wiesbaden: Westdeutscher Verlag, 104-131.

Wu, H. Denis (2000): Systemic determinants of international news coverage: a comparison of 38 countries. In: Journal of Communication 50 (1): 110-130.

Zöllner, Oliver (2002): Deutsches Auslandsfernsehen: Akzeptanz von DW-TV. Ergebnisse der internationalen Nutzungsforschung. In: Media Perspektiven 5: 232-238. 